\title{
Focal necrotising herpes simplex encephalitis: a report of two cases with good clinical and neuropsychological outcomes
}

\author{
C E Counsell, R Taylor, I R Whittle
}

\begin{abstract}
Two patients with atypical focal necrotising herpes simplex encephalitis are described. They presented with relatively mild clinical disease but despite treatment with acyclovir (10 $\mathrm{mg} / \mathrm{kg} / \mathrm{day}$ for three days in case 1 and 10 days in case 2) they developed dramatic, progressive changes (shown on brain CT) that mimicked space occupying lesions of the temporal lobes. Both patients therefore had a diagnostic and therapeutic temporal lesionectomy followed by further 10 day courses of acyclovir. They subsequently went on to make good clinical and neuropsychological recoveries.
\end{abstract}

$(\mathcal{F}$ Neurol Neurosurg Psychiatry 1994;57:1115-1117)

Herpes simplex encephalitis (HSE) typically presents as an acute pyrexial illness associated with altered conscious level, confusion, behavioural changes, seizures, and focal symptoms and signs related to preferential involvement of the temporal lobes. ${ }^{1}$ Acyclovir has been shown to dramatically reduce the mortality, from around $70 \%$ to $20 \%{ }^{2}$ Considerable neurological morbidity remains, however-up to $30 \%$ in one series ${ }^{2}$ - with this being highest in patients with necrotising encephalitis.

We report two cases of atypical focal necrotising HSE who presented with relatively mild symptoms but had dramatic progressive focal changes on brain CT, which caused diagnostic confusion. Both made very good recoveries after surgical intervention.

Department of Clinical Neurosciences, Western General Hospital, Edinburgh EH4 2XU, UK C E Counsell $\mathrm{R}$ Taylor

I R Whittle

Correspondence to:

Dr C E Counsell,

Neurosciences Trials Unit, Department of Clinical

Neurosciences, Western

General Hospital,

Edinburgh EH4 2XU, UK.

Received 4 October 1993

and in revised form

8 February 1994.

Accepted 22 February 1994 showed a normal full blood count, urea, electrolytes, and glucose and a normal chest radiograph. Three sets of blood cultures were all negative and CT of the brain was normal. Examination of CSF showed 750 white cells $/ \mathrm{mm}^{3}$, predominantly lymphocytes, a raised protein concentration $(0.83 \mathrm{~g} / \mathrm{l})$, and a normal glucose. There was no growth on culture.

A diagnosis of possible HSE was made and he was started on acyclovir (10 mg/ $/ \mathrm{kg} /$ day). At neurological review three days later, it was considered that he was too well to have HSE and therefore the acyclovir was stopped. $\mathrm{He}$ continued to have a mild headache and low grade fever and subsequently had two generalised tonic-clonic seizures. A repeat brain CT showed an area of low density in the right frontotemporal area and repeat CSF examination showed a persistent lymphocytosis of greater than 700 cells $/ \mathrm{mm}^{3}$ and a protein concentration of $1.3 \mathrm{~g} / 1$. An EEG showed widespread abnormalities with sharp complexes on the right compatible with HSE. Ten days after admission he developed a partial right third nerve palsy with pupil involvement and became slightly drowsy (Glasgow coma scale 14). Repeat brain CT showed a mixed low and high attenuation lesion in the right temporal lobe with some midline shift and ring enhancement (figure (A)). This raised the possibility of a subdural empyaema, a cerebral abscess, or necrotising encephalitis. He was therefore started on intravenous antibiotics and dexamethasone and transferred to the neurosurgical unit.

The next day he had a craniotomy, which showed a necrotic right temporal lobe with multiple cortical haemorrhages. A piecemeal temporal lobectomy was performed of all three temporal gyri plus the amygdala and hippocampus. Histology showed necrotic brain tissue with pronounced lymphocytic infiltration and occasional nuclear inclusion bodies. Immunocytochemistry for herpes simplex type 1 virus was positive (figure (B)) and herpes simplex type 1 virus was isolated from the tissue. After operation, a complete 10 day course of acyclovir was given and the patient made an uncomplicated recovery.

Three months after the operation he was back to his normal job with no complaints. At one year the patient considered that he had made a full recovery. He had experienced no 
(A) Brain CT of patient 1. Precontrast (left) there is expansion of the right medial temporal lobe with diffuse low density and a focal high density lesion suggestive of $a$ haemorrhage, and right to left shift. After contrast (right) (45 ml niopam) there is peripheral enhancement (single large arrow), and the uncal herniation can be clearly seen (double small arrows). (B)

Immunocytochemistry for herpes simplex type 1 virus with a polyclonal antibody (Dako, UK) shows positive reactions in neurons and astrocytes, and widespread inflammation is evident haematoxylin counterstain; origina magnification 250).

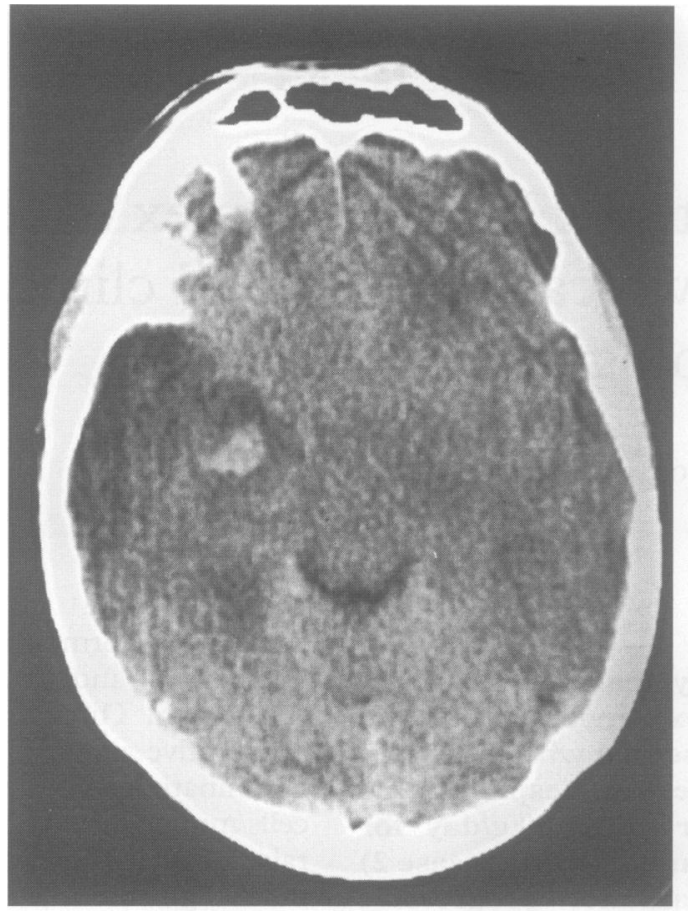

A

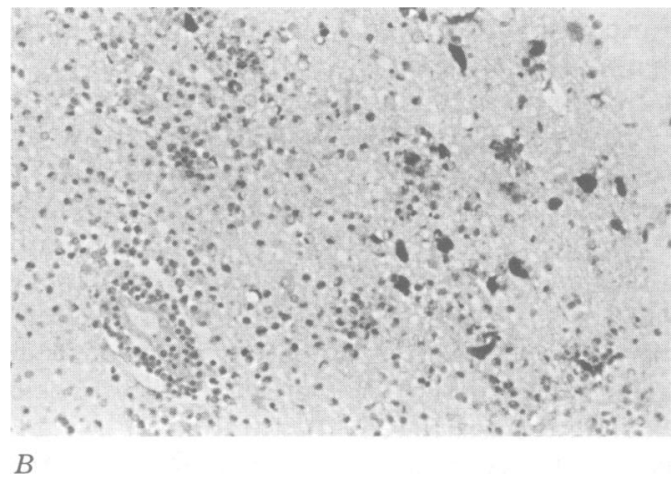

difficulties at his work, and was playing football and rock climbing.

CASE 2

A 14 year old right handed schoolboy was admitted to his local hospital with a 10 day history of fever, headaches, and lethargy. On the day of admission his father had witnessed a generalised tonic-clonic seizure. Examination was unremarkable except for a pyrexia and slight drowsiness. Examination of CSF showed 600 white cells $/ \mathrm{mm}^{3}$, mainly lymphocytes, a protein concentration of $0.98 \mathrm{~g} / \mathrm{l}$, a normal glucose, and no growth on culture. He was transferred to our unit for a brain CT.

On arrival he was drowsy (Glasgow coma scale 14) and had a temperature of $39^{\circ} \mathrm{C}$, but was otherwise well. He had early bilateral papilloedema and a drift of his left arm. Brain CT showed an ill defined area of low attenuation in the right temporal lobe with some mass effect. An EEG showed irregular slowing in the right hemisphere with occasional sharp waves in the temporal region.

A diagnosis of HSE was made and he received a full 10 day course of acyclovir. $\mathrm{He}$ improved but continued to have an intermit-

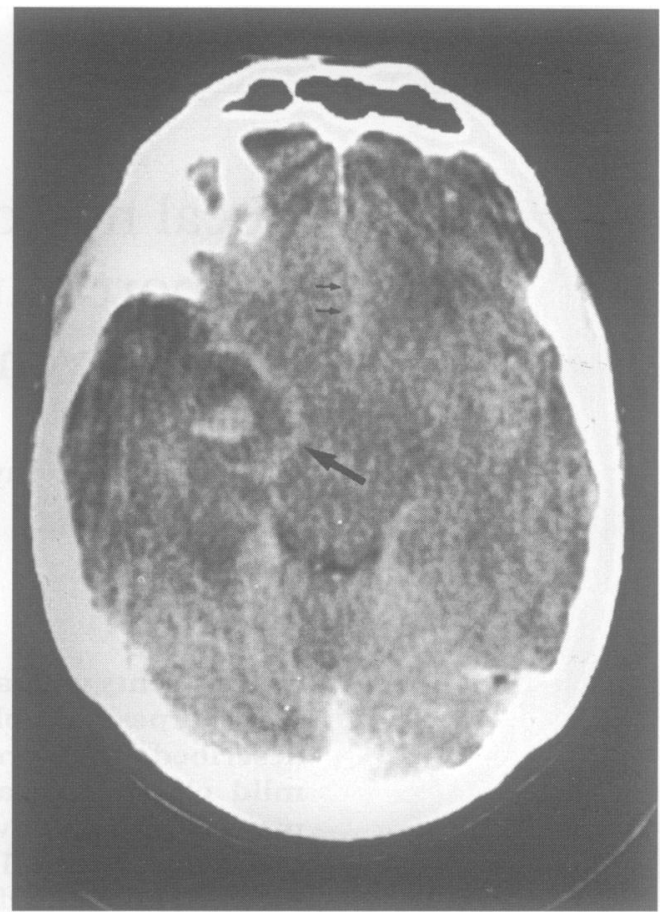

tent pyrexia. On stopping the acyclovir, however, the headache returned, he became slightly vague, and his papilloedema was worse. A further brain CT showed that the lesion in the right temporal lobe had increased in size and was now enhanced after intravenous contrast. The possibility of cerebral lymphoma was raised and a biopsy was arranged.

At craniotomy the superior temporal gyrus was noted to be necrotic. A frozen section was initially reported as showing "a small cell malignancy", possibly from a primary neuroectodermal tumour, and therefore a temporal lobectomy was performed although the amygdala and hippocampus were not approached. Formal histology showed an extensive necrotising process involving grey and white matter with an intense lymphocytic infiltrate, and no evidence of malignancy. Herpes simplex virus was not cultured from either the CSF or brain biopsy but there was a greater than fourfold increase in the serum antibody titres to the virus over the course of the illness (from less than 16 to greater than 256) and subsequent immunocytochemistry for herpes simplex type 1 virus was positive.

After operation he received a further 10 day course of acyclovir and made a rapid recovery. By two months he was back to school and according to him and his parents was "back to normal in every way". At one year he had no abnormal neurological signs and according to his teachers was performing well at school with no deterioration in his academic performance since his illness.

\section{Neuropsychological assessments}

Both patients had brief psychological assessments one week after surgery. At this time the first patient showed mild psychomotor slowing and impairments in attention and 
concentration, constructional ability, and non-verbal memory. The second patient showed only some non-verbal memory impairment in recalling the Rey complex figure.

Subsequently, both patients had extensive psychological testing with the following: National audit reading test; WAIS-R; Warrington recognition memory tests for words and faces; paragraph recall; copy and recall of the Rey complex figure; language screening tests covering comprehension, repetition, naming, reading, and writing; a test of motor praxis; perceptual screening tests; Hooper visual organisation test items; Wisconsin card sorting; generation of words in given categories; and the cognitive estimation test. The younger patient's performances were compared with the youngest adult norms, which would tend to lead to an underestimation of his abilities.

The first patient, assessed only two months after the onset of his illness, had no evidence of intellectual deterioration compared with his premorbid estimate. Language was normal and calculation excellent. He performed normally or above average on all tests except for the WAIS-R object assembling and picture arrangement subtests and copying the Rey complex figure, where he performed slightly below average, and recall of the Rey complex, where his performance was in the definitely impaired range. One month later there had been further improvement and he returned to work as a computer consultant shortly afterwards.

The second patient, assessed 15 months after his illness, performed normally or better than average on all tests and subtests except that on the Warrington memory tests his memory for faces was only average and not as good as his memory for words. His talents in musical composition and performance were apparently undiminished. He was doing well at school and was planning to go to university.

\section{Discussion}

These two cases of focal necrotising HSE show a distinct clinical course. Both presented with a short history suggestive of HSE and both improved with acyclovir (although the first patient did not have a complete course). There was a persistent low grade fever and when the acyclovir was stopped there was some clinical deterioration. This has been reported previously, even after a full 10 day course of acyclovir. ${ }^{3}$ By contrast with their relatively stable clinical state, however, they developed progressive focal changes on brain CT, mimicking space occupying lesions. The degree and pattern of contrast enhancement of these lesions were unusual. Previous reports of CT in patients with HSE have highlighted the low attenuation focal lesions that can be associated with a pronounced mass effect and some enhancement $^{4-6}$ but the enhancement is usually linear or gyral rather than the peripheral ring enhancement seen in our cases. Both our cases made very good recoveries after surgical intervention with little in the way of physical or psychological morbidity.

It is possible that these two presentations are the result of acyclovir modifying the natural course of the disease. Alternatively they may represent a rare but specific pattern of infection. The good outcome in both patients is likely to relate partly to the facts that the patients were young and the disease remained localised to the non-dominant hemisphere. We also believe that early neurosurgical intervention with removal of the necrotic mass lesion may have played a part in minimising the morbidity.

In summary, focal necrotising HSE can mimic a space occupying lesion and may require treatment with a prolonged course of acyclovir (to be continued as long as clinical signs indicate, together perhaps with a repeat CT) plus neurosurgical intervention where appropriate. This can result in a very good clinical outcome.

We are grateful to $\mathrm{Dr} J \mathrm{~W}$ Ironside, Neuropathology Laboratory, Western General Hospital, Edinburgh for reviewing the histology and providing the illustration of the immunocytochemistry.

1 Whitley RJ, Soong S-J, Linneman C, Liu C, Pazin G, Alford CA, and the National Institute of Allergy and Infectious Diseases Collaborative Antiviral Study Group. Herpes simplex encephalitis. Clinical assessment. $\mathcal{F} A M A$ 1982;247:317-20.

2 Skoldenberg B, Alestig K, Burman A, et al. Acyclovir versus vidarabine in herpes simplex encephalitis. Randomised multicentre study in consecutive Swedish patients. Lancet 1984;2:707-11.

3 VanLandingham KE, Marstellar HB, Ross GW, Hayden FG. Relapse of herpes simplex encephalitis after conventional acyclovir the, $\mathcal{A} A M A$ 1988;259:1051-3.

4 Davis JM, Davis KR Kleinman GM, Kirchner HS Taveras JM. Computed tomography of herpes simplex Radiology 1978;129:409-17.

5 Enzmann DR, Ranson B, Norman D, Talberth E. Computed tomography of herpes simplex encephalitis. Radiology 1979;129:419-25.

6 Kaufman DM, Zimmerman RD, Leeds NE. Computed tomography in Herpes simplex encephalitis. Neurology 1979;29:1392-6. 